\title{
Contents
}

\section{Part I Nanowires}

From Ordered Arrays of Nanowires to Controlled Solid State Reactions

Margit Zacharias and Hong Jin Fan......................... 3

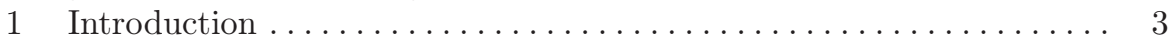

2 Methods for nano-patterning ....................... 6

3 Selected results - stimulated emission $\ldots \ldots \ldots \ldots \ldots \ldots \ldots .7$

4 Nanotubes based on Kirkendall diffusion and solid state reactions . . 8

$5 \quad$ Summary .................................. 11

6 Acknowledgement ............................... 12

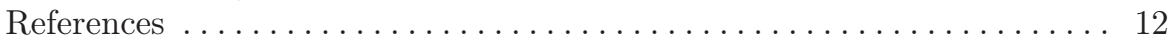

Growth Methods and Properties of High Purity III-V

Nanowires by Molecular Beam Epitaxy

D. Spirkoska, C. Colombo, M. Heiß, M. Heigoldt, G. Abstreiter, and

A. Fontcuberta i Morral ................................ 13

1 Introduction ................................... 13

2 Experimental .................................. 14

3 Selective area epitaxy ............................ 14

4 Conditions leading to group III assisted growth of nanowires ...... 17

5 Structural and optical properties . ..................... 19

6 Potential for future structures and devices ................ 21

7 Conclusions.................................. 23

8 Acknowledgements .............................. 23

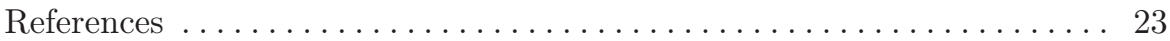

Simple Ways to Complex Nanowires and Their Application Mady Elbahri, Seid Jebril, Sebastian Wille, and Rainer Adelung....... 27

1 Introduction ................................. 27

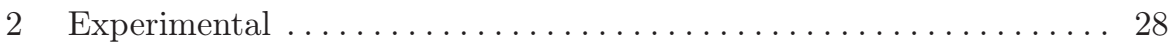




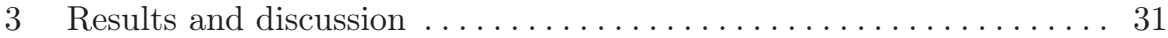

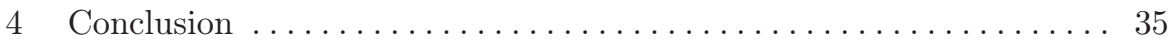

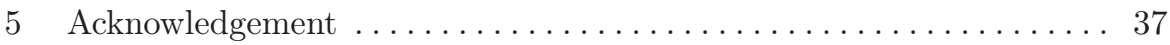

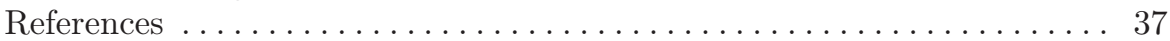

ZnO Nanostructures: Optical Resonators and Lasing

Klaus Thonke, Anton Reiser, Martin Schirra, Martin Feneberg,

Günther M. Prinz, Tobias Röder, Rolf Sauer, Johannes Fallert,

Felix Stelzl, Heinz Kalt, Stefan Gsell, Matthias Schreck, and

Bernd Stritzker .................................. 39

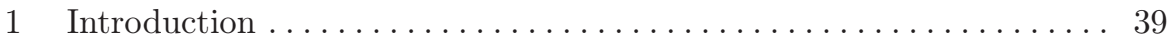

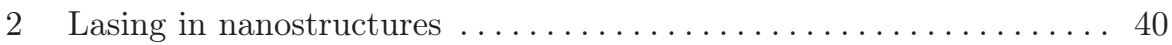

3 Single $\mathrm{ZnO}$ pillars as nano-resonators $\ldots \ldots \ldots \ldots \ldots \ldots \ldots \ldots \ldots 4$

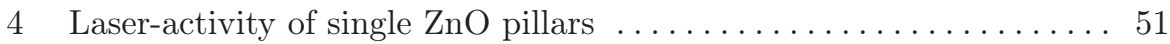

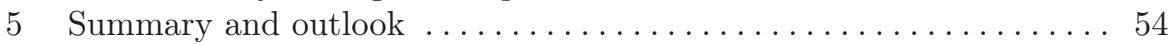

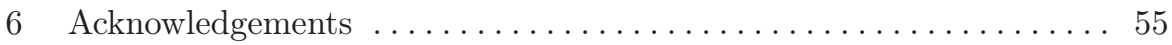

References ................................. 55

\section{Waveguiding and Optical Coupling in $\mathrm{ZnO}$ Nanowires and Tapered Silica Fibers}

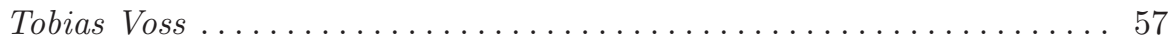

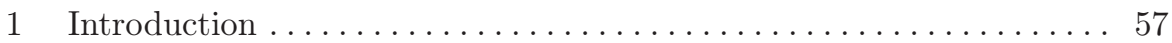

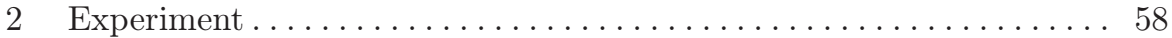

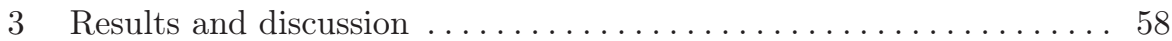

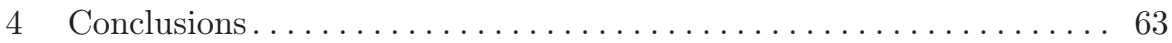

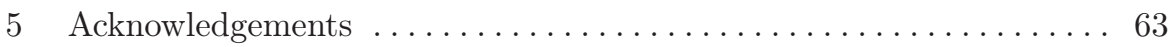

References ............................... 64

\section{Part II Quantum Dots and Nanoparticles}

\section{Electrically Driven Single Quantum Dot Emitter Operating} at Room Temperature

Tilmar Kümmell, Robert Arians, Arne Gust, Carsten Kruse, Sergey

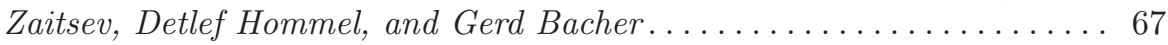

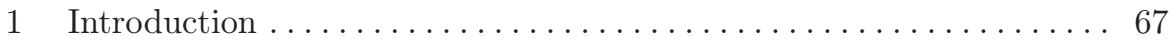

2 Quantum dots optimized for RT emission................. 68

3 Electrically driven single quantum dot emitter $\ldots \ldots \ldots \ldots \ldots \ldots 73$

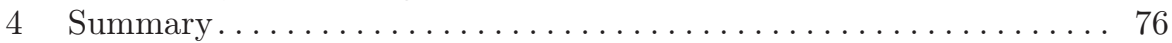

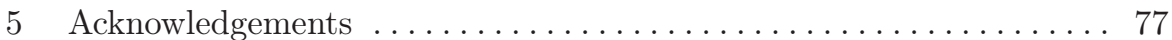

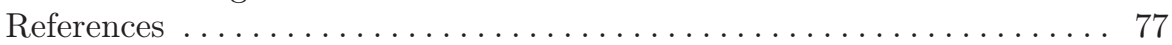


Silicon Nanoparticles: Excitonic Fine Structure and Oscillator Strength

Cedrik Meier, Stephan Lüttjohann, Matthias Offer, Hartmut Wiggers, and Axel Lorke . . . . . . . . . . . . . . . . . . . . . . . . . . . . . 79

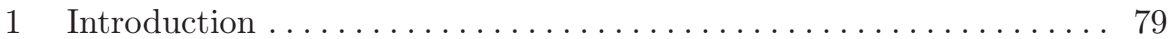

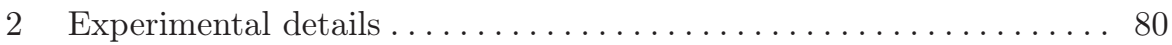

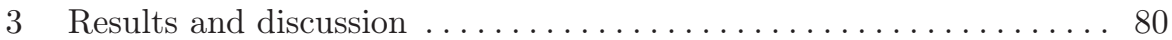

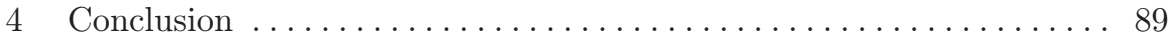

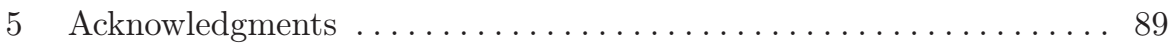

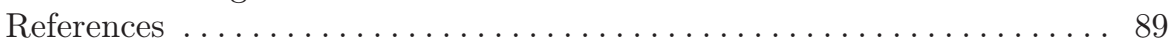

Intrinsic Non-Exponential Decay of Time-Resolved Photoluminescence from Semiconductor Quantum Dots

Jan Wiersig, Christopher Gies, Norman Baer, and Frank Jahnke . . . . . 91

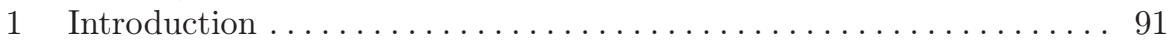

2 Time-resolved photoluminescence ................... 92

3 Correlations . . . . . . . . . . . . . . . . . . . . . . . . . . . . 95

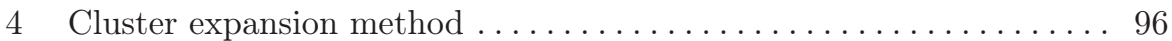

5 Numerical results: non-exponential PL decay . . . . . . . . . . . . 97

6 Numerical results: excitation-intensity dependence . . . . . . . . . . . 99

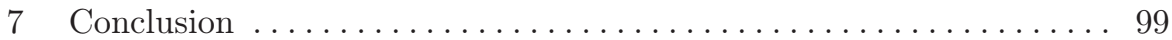

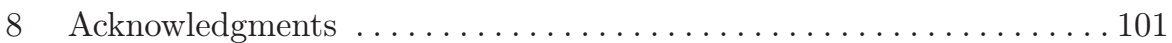

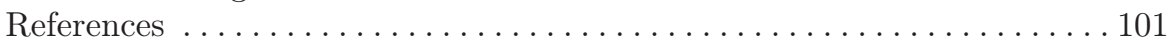

Electrical Spin Injection into Single InGaAs Quantum Dots

Michael Hetterich, Wolfgang Löffler, Pablo Aßhoff, Thorsten Passow, Dimitri Litvinov, Dagmar Gerthsen, and Heinz Kalt. . . . . . . . . . . . 103

1 Introduction . . . . . . . . . . . . . . . . . . . . . . . . . 103

2 Initialization and readout of spins in single dots . . . . . . . . . . 105

3 Spin loss mechanisms and device optimization . . . . . . . . . . . 107

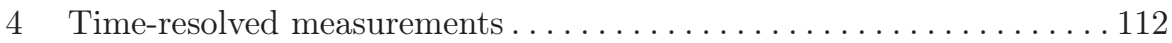

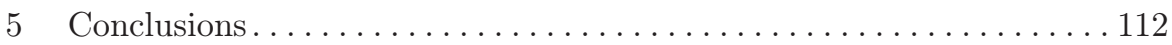

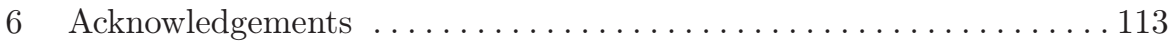

References .................................... 113

\section{Part III Spin and Magnetism}

\section{Spintronic and Electro-Mechanical Effects in Single-Molecule} Transistors

Maarten R. Wegewijs, Felix Reckermann, Martin Leijnse, and Herbert

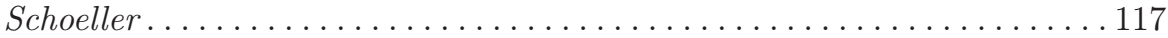

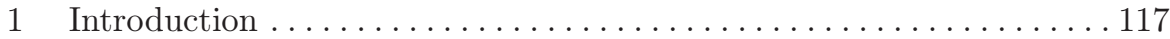

2 Mixed-valence dimer transistor . . . . . . . . . . . . . . . . . . . . . . . . . . 119

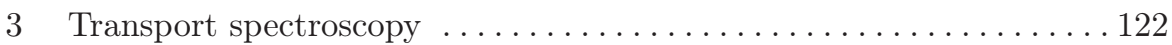

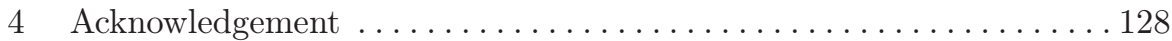

References .................................... 128 
Transport in 2DEGs and Graphene: Electron Spin vs. Sublattice Spin

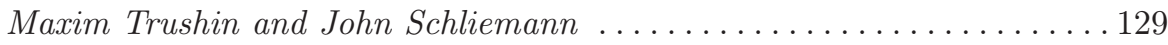

1 Introduction . . . . . . . . . . . . . . . . . . . . . . . . . . . . 129

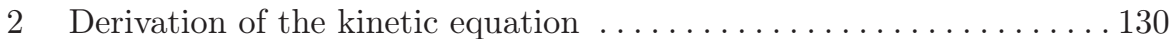

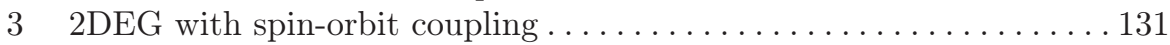

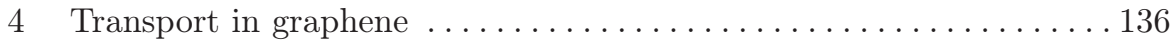

5 Conculsion remarks . . . . . . . . . . . . . . . . . . . . . . 139

References .................................... 140

\section{Spin Dynamics in High-Mobility Two-Dimensional Electron} Systems

Tobias Korn, Dominik Stich, Robert Schulz, Dieter Schuh, Werner

Wegscheider, and Christian Schüller.................... 143

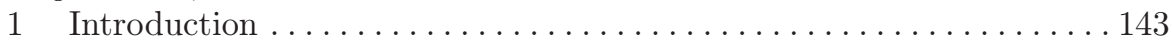

2 Theory................................. 144

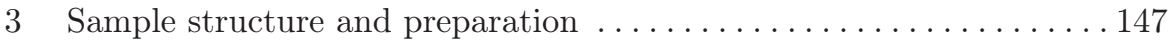

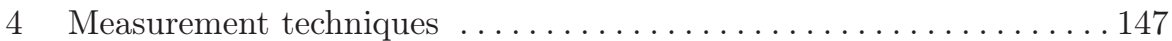

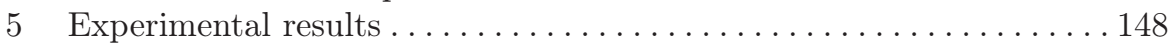

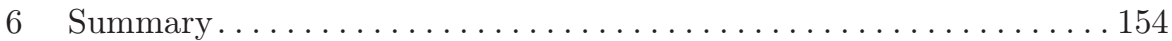

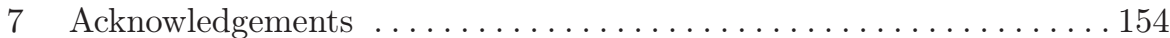

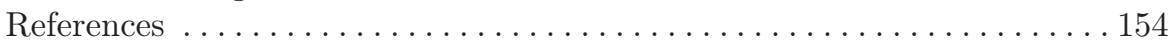

Magnetization Dynamics of Coupled FerromagneticAntiferromagnetic Thin Films

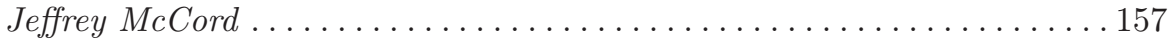

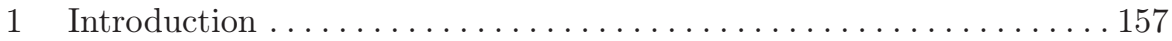

2 Magnetization dynamics. . . . . . . . . . . . . . . . . . 158

3 Statics and dynamics of exchange biased $\mathrm{F} / \mathrm{AF}$ films . . . . . . . . . 160

$4 \mathrm{~F} / \mathrm{AF} / \mathrm{F}$ structures below the onset of exchange bias . . . . . . . . . 163

5 Conclusions..................................... 168

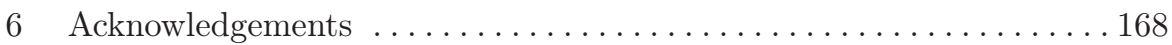

References ...................................... 168

\section{Magnetic and Electronic Properties of Heusler Alloy Films}

Investigated by X-Ray Magnetic Circular Dichroism

Hans-Joachim Elmers, Andres Conca, Tobias Eichhorn, Andrei

Gloskovskii, Kerstin Hild, Gerhard Jakob, Martin Jourdan, and

Michael Kallmayer.................................171

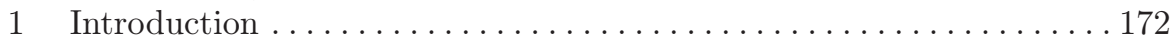

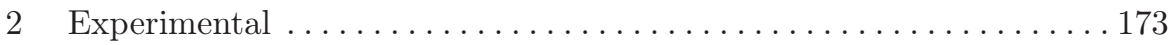

3 Martensitic phase transition in $\mathrm{Ni}_{2} \mathrm{MnGa}$ films . . . . . . . . . . . . 173

4 Interface properties of Heusler compound films . . . . . . . . . . . . . . . . 177

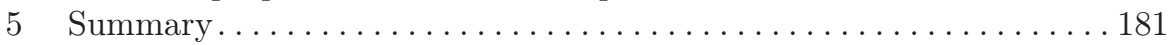

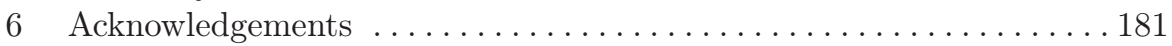

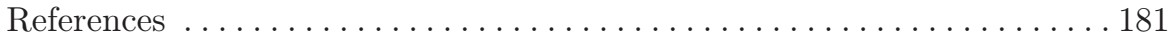


Coherent Spin Dynamics in Nanostructured SemiconductorFerromagnet Hybrids

Patric Hohage, Jörg Nannen, Simon Halm, and Gerd Bacher ......... 183

1 Introduction . . . . . . . . . . . . . . . . . . . . . 183

2 Samples and experiment .......................... 184

3 Free vs. localized spin precession in a semiconductor . . . . . . . . . 185

4 Spin dynamics in ferromagnet-semiconductor hybrids . . . . . . . . . 187

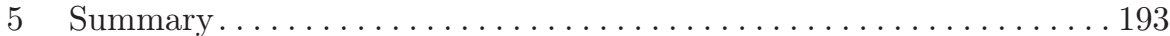

6 Acknowledgements . . . . . . . . . . . . . . . . . . . . . 194

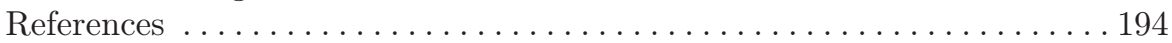

\section{Part IV Organic Materials and Water}

\section{Coupling of Paramagnetic Biomolecules to Ferromagnetic Surfaces}

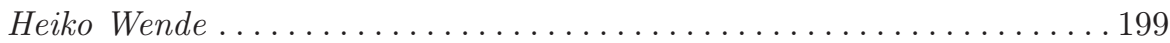

1 Introduction . . . . . . . . . . . . . . . . . . . . . . . . . . . 199

2 Experimental details .............................. 200

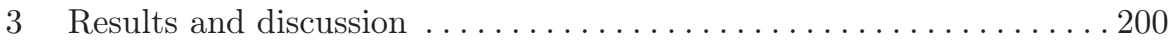

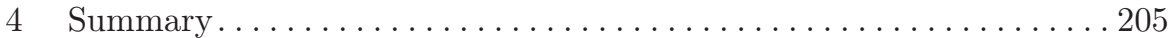

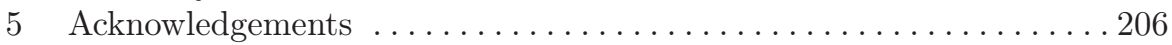

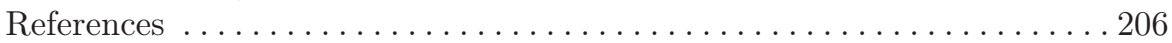

\section{Band Alignment in Organic Materials}

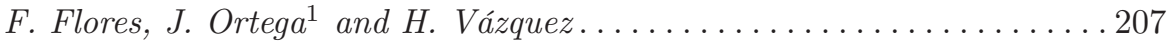

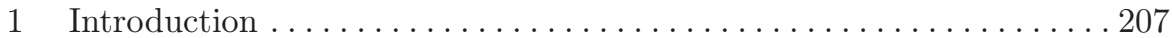

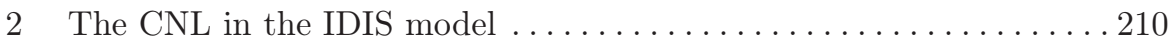

3 The IDIS model at MO interfaces $\ldots \ldots \ldots \ldots \ldots \ldots \ldots \ldots \ldots \ldots \ldots \ldots$

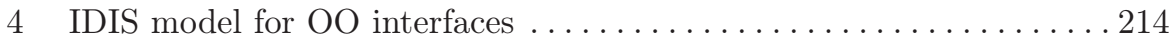

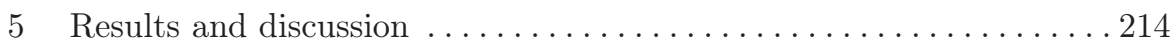

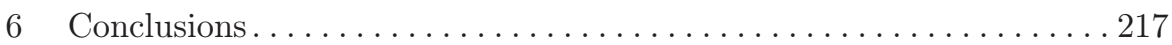

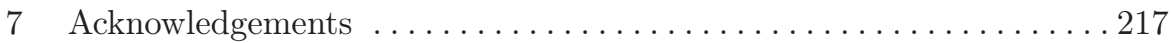

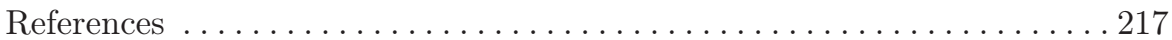

Organometallic Nanojunctions Probed by Different

Chemistries: Thermo-, Photo-, and Mechano-Chemistry

Martin Konôpka, Robert Turanský, Nikos L. Doltsinis, Dominik Marx, and Ivan Śtich . . . . . . . . . . . . . . . . . . . . . . . . . . . . . . . . . . . . . . . . . 219

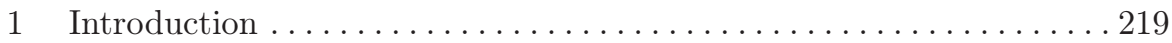

2 Thermo- and Mechano-chemistry of copper-ethylthiolate junctions . . 220

3 Mechanically and opto-mechanically controlled azobenzene (AB) switch based on AB-gold break-junction. . . . . . . . . . . . . . 225

4 Conclusions... . . . . . . . . . . . . . . . . . . . . . . . . . . . . . . 231

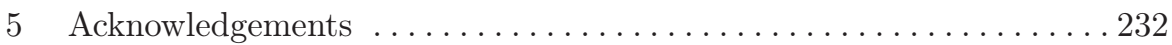

References ........................................ 233 
When It Helps to Be Purely Hamiltonian: Acceleration of Rare Events and Enhanced Escape Dynamics

Dirk Hennig, Simon Fugmann, Lutz Schimansky-Geier, and

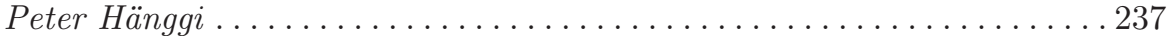

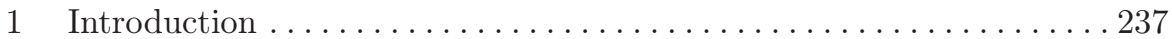

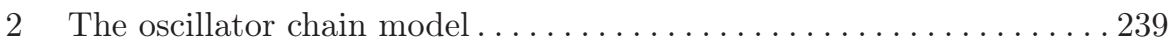

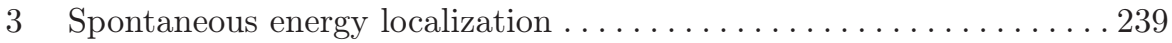

4 Escape dynamics . . . . . . . . . . . . . . . . . . . . . . . . . 242

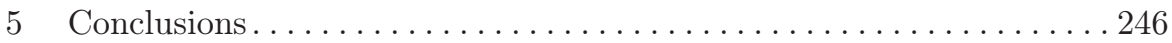

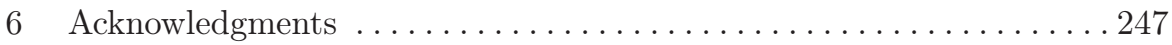

References ................................... 247

Liquid Polyamorphism and the Anomalous Behavior of Water

H. E. Stanley, S. V. Buldyrev, S.-H. Chen, G. Franzese, S. Han,

P. Kumar, F. Mallamace, M. G. Mazza, L. Xu, and Z. Yan ......... 249

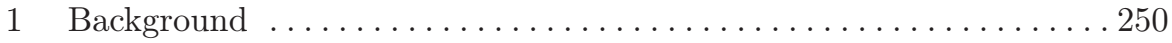

2 Understanding "static heterogeneities" . . . . . . . . . . . . . . . . . . 252

3 Understanding "dynamic heterogeneities" . . . . . . . . . . . . . . . . 257

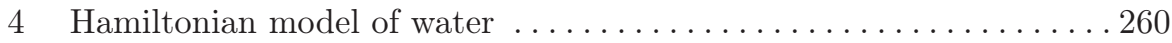

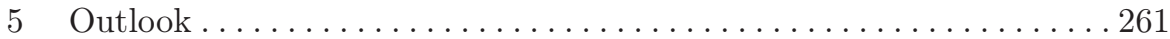

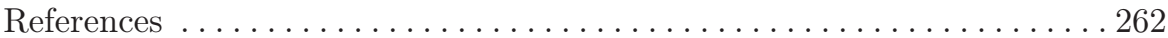

\section{Part V Dynamical Effects, Rectification and Nonlinearities}

Terahertz Detection of Many-Body Signatures in Semiconductor Heterostructures

Sangam Chatterjee, Torben Grunwald, Stephan W. Koch, Galina

Khitrova, Hyatt M. Gibbs, and Rudolf Hey .................. 269

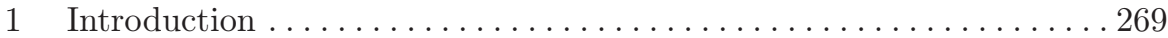

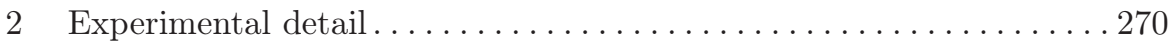

3 Data analysis . . . . . . . . . . . . . . . . . . . 272

4 Intra-excitonic 1s-2p transition in GaAs/(AlGa)As quantum wells . . 274

5 Intra-excitonic 1s-2p transition in (GaIn)As/GaAs quantum wells . . 276

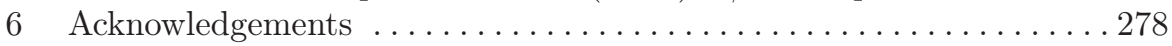

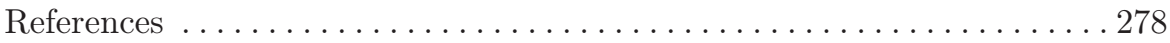

Theory of Ultrafast Dynamics of Electron-Phonon Interactions in Two Dimensional Electron Gases: Semiconductor Quantum Wells, Surfaces and Graphene

Marten Richter, Stefan Butscher, Norbert Bücking, Frank Milde,

Carsten Weber, Peter Kratzer, Matthias Scheffler, and Andreas Knorr . 281

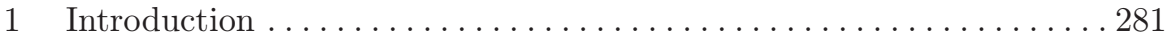

2 Theoretical framework . . . . . . . . . . . . . . . . . . 282

3 Phonon-induced relaxation dynamics at the silicon (001) $2 \times 1$ surface . . . . . . . . . . . . . . . . . . . . . . . . . . . . . . . 284 
4 Scattering response and spatiotemporal wavepackets in quantum cascade lasers . . . . . . . . . . . . . . . . . . . . . . . . 286

5 Non-equilibrium phonon dynamics in graphene . . . . . . . . . . . 287

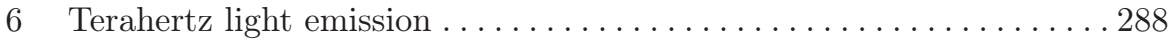

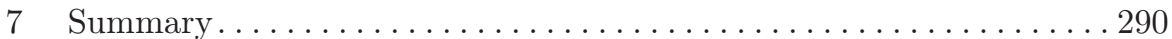

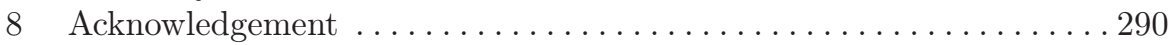

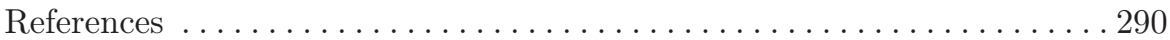

\section{Optical Microcavities as Quantum-Chaotic Model Systems:} Openness Makes the Difference!

Martina Hentschel . . . . . . . . . . . . . . . . . . . . . . . . . . . . . . . . 293

1 Introduction . . . . . . . . . . . . . . . . . . . . . . . . . . . 293

2 Deviations from ray-wave correspondence. . . . . . . . . . . . . . . . 297

3 Correcting ray optics by wave effects: Goos-Hänchen shift and Fresnel filtering . . . . . . . . . . . . . . . . . . 300

4 Outlook: non-Hamiltonian dynamics in quantum-chaotic model

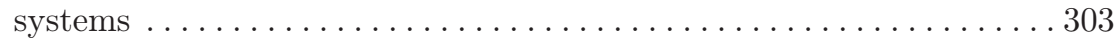

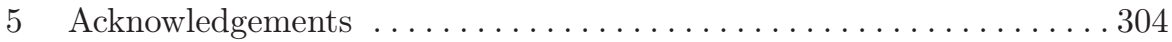

References ...................................... 304

Nonlinear Transport Properties of Electron Y-Branch Switches

Lukas Worschech, David Hartmann, Stefan Lang, D. Spanheimer,

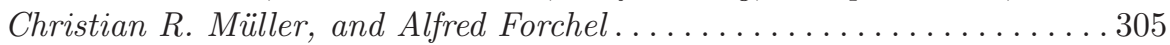

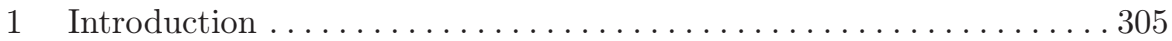

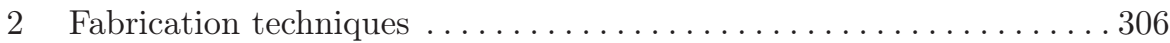

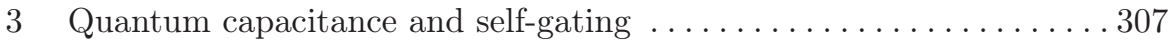

4 Self-gating in a Y-branch switch at room temperature . . . . . . . . . 308

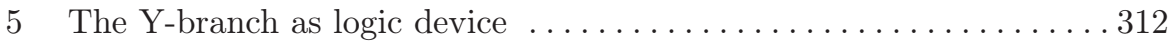

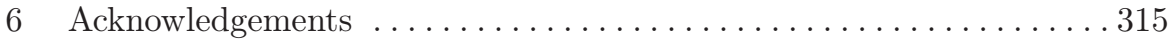

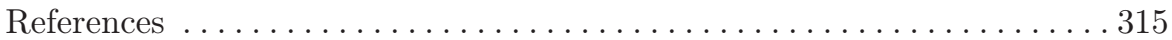

Rectification through Entropic Barriers

Gerhard Schmid, P. Sekhar Burada, Peter Talkner, and Peter Hänggi . . 317

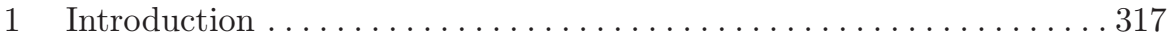

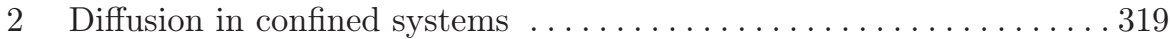

3 Transport in periodic channels with broken symmetry . . . . . . . . . 320

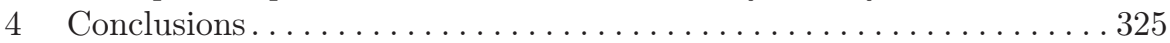

5 Acknowledgements . . . . . . . . . . . . . . . . . . . . . . . . 326

References ................................ 326 


\section{Part VI Characterization of Materials and Devices}

Microstructure Tomography - An Essential Tool to Understand 3D Microstructures and Degradation Effects

Alexandra Velichko and Frank Mücklich . . . . . . . . . . . . . . . . . . . 331

1 Introduction . . . . . . . . . . . . . . . . . . . . . . . . . . . 331

2 Basic characteristics of the microstructure . . . . . . . . . . . . 332

3 Determination of the $3 \mathrm{D}$ grain size distribution from $2 \mathrm{D}$

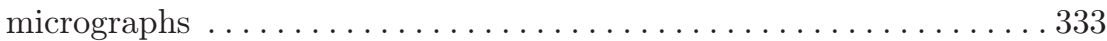

4 Analysis of the 3D tomographical images . . . . . . . . . . . . 335

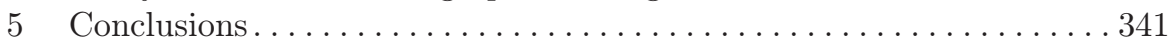

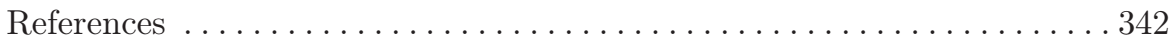

\section{Profiling of Fiber Texture Gradients by Anomalous X-ray Diffraction}

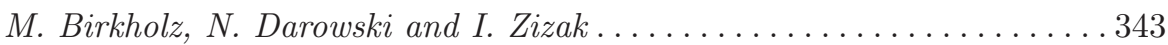

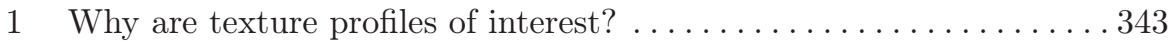

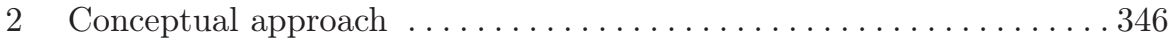

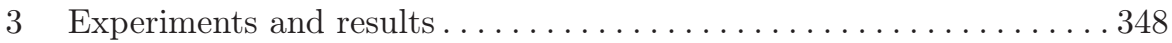

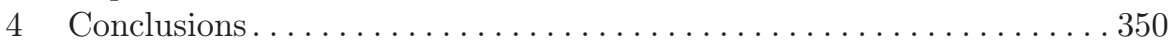

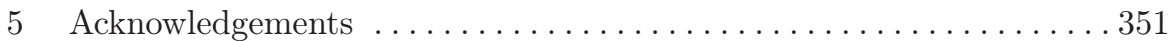

References ...................................... 351

Film Production Methods in Precision Optics

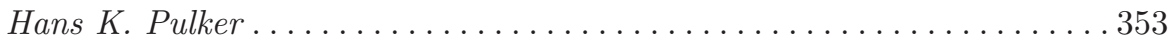

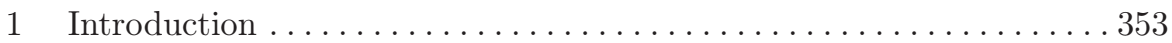

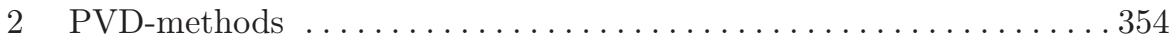

3 Thin film properties ............................ 364

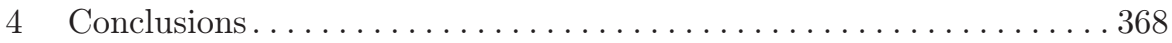

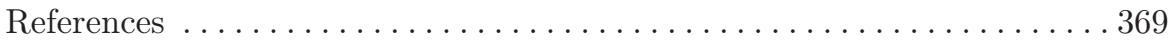

Advanced Metrology for Next Generation Transistors

Alain C. Diebold .................................. 371

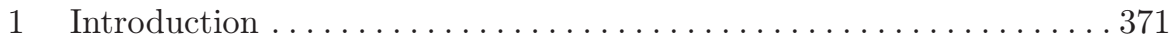

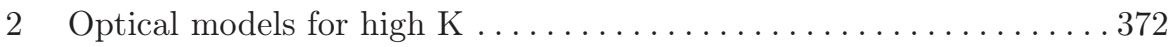

3 Optical model for metal films . . . . . . . . . . . . . . . . . . 377

4 Charge pumping based capacitance - voltage measurements . . . . . . 377

5 Optical measurement of charge trapped in high $\mathrm{k}$ and interface . . . . 378

6 Measurement of ultra-thin SOI and observation of quantum confinement . . . . . . . . . . . . . . . . . . . . . . . . . . . 380

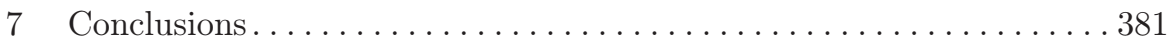

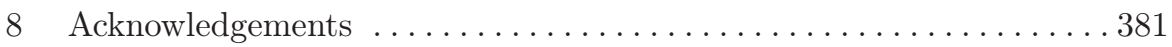

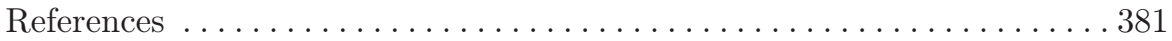

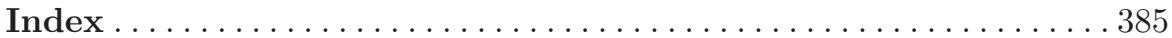

